\title{
Harmonic Impact analysis coming from the manufacturing processes of a Eletroeletrônica Industry Using KDD and Decision Trees
}

\author{
${ }^{1}$ Rildo de Mendonça Nogueira, ${ }^{1}$ Edson Farias de Oliveira, ${ }^{1}$ Waterloo Ferreira da Silva, ${ }^{2}$ Ádamo L. Santana, ${ }^{2}$ Maria Emília \\ de Lima Tostes, ${ }^{2}$ Thiago Mota Soares \\ 1 Programa de Pós Graduação em Engenharia Elétrica - PPGEE, Universidade Federal do Pará - UFPA \\ Belém - PA, Brasil \\ 2 Instituto de Tecnologia - ITEC, Universidade Federal do Pará - UFPA \\ Belém - PA, Brasil \\ rildonogueira,edsonfarias2007,waterloof\}@hotmail.com,tostes,adamo,thiagosoares\}@ufpa.br
}

\begin{abstract}
This paper approaches the analysis of the current distortion impact due the harmonic frequencies present at the power grid of an industry facility at Manaus during the manufacturing process of informatics devices. The reason for such frequencies to appear are the presence of non linear loads from personal computers, notebooks and tablets. The data were collected in a measurement campaign during a week, using quality energy analyzers at different production process supplied by $13,8 \mathrm{kV}$ and with a nominal load of $800 \mathrm{kVA}$. The main objective of this paper is to present an analysis based on computational intelligence using decision tree to determine and quantify the harmonic distortions commonly present at manufacturing process of informatics industries using simultaneous measurements of voltage and current to determine which process has the biggest impact in the total harmonic distortion at the common coupling point between the factory and the energy dealers.
\end{abstract}

Keywords: Energy Quality, Total Harmonic Distortion of Voltage, Total Harmonic Distortion of Individual Current, Decision Tree.

\section{Analises De Impactos Harmônicos Oriundos Dos Processos De Manufatura De Uma Indústria De Eletroeletrônica Utilizando Kdd E Árvores De Decisão}

\section{RESUMO}

O trabalho aborda a análise do impacto de distorção atual, devido as freqüências harmônicas presentes na rede de energia de uma instalação de indústria em Manaus durante o processo de dispositivos de informática de fabricação. A razão para essas frequências para aparecer são a presença de cargas não lineares a partir de computadores pessoais, notebooks e tablets. Os dados foram coletados em uma campanha de medição durante uma semana, usando analisadores de energia de qualidade a diferentes processos de produção fornecidos pela $13,8 \mathrm{kV}$ e com uma carga nominal de $800 \mathrm{kVA}$. O principal objetivo deste trabalho é apresentar uma análise baseada em inteligência computacional utilizando árvore de decisão para determinar e quantificar as distorções harmônicas comumente presentes no processo de fabricação de indústrias de informática usando medições simultâneas de tensão e corrente para determinar qual processo tem o maior impacto no distorção harmônica total no ponto de acoplamento comum entre a fábrica e os concessionários de energia.

Palavras Chaves: Qualidade no processo, Soldagem Reflow; Componente BGA, Cross section, Cracks.

\section{INTRODUÇÃO}

O termo genérico eletrônica de potência é empregado nos sistemas elétricos e nos produtos que envolvem controle e conversão do fluxo de energia elétrica. Atualmente, os sistemas elétricos sofrem grandes influências na geração de fontes contaminantes ou produtoras de harmônica destacando-se fundamentalmente, as cargas não lineares empregadas nas instalações industriais, residências e comerciais. Com isto, 
Rildo de Mendonça Nogueira, Edson Farias de Oliveira, et al / ITEGAM-JETIA Vol.01, Nº 01, pp.67-74. Março, 2015.

contribui-se para o aumento de problemas relacionados com a qualidade da energia elétrica fornecida [1][2].

Por outro lado, as cargas monofásicas não lineares e de baixa potência que possuem alta representatividade na geração de distorções harmônicas e, que estão presentes em grande número, principalmente em aparelhos eletrônicos, fontes de alimentação, notebooks, reatores de lâmpadas fluorescentes, monitores de vídeos, tablets e computadores de mesa. As consequências mais significativas das distorções harmônicas são detectadas no Ponto de Acoplamento Comum - PAC, neste ponto as cargas especificas e outras cargas consumidoras do sistema elétrico de potência estão ligadas em um ponto comum com a concessionária de energia elétrica [3].

As medições das distorções harmônicas têm sido usadas para caracterizar o comportamento de cargas não lineares, para localizar fontes harmônicas e quantificar os níveis de distorções harmônicas em sistemas de energia [4]. Mesmo em novos conceitos de distribuição de energia elétrica, há uma preocupação com a distorção harmônica total (THD) e outros indicadores de qualidade de energia [5]. O THD é o índice harmônico mais comum usado para avaliar e medir as condições de variações da Qualidade da Energia em condições não senoidais.

No Brasil, a Agência Nacional de Energia Elétrica (ANEEL) desenvolveu os Procedimentos de Distribuição de Energia (PRODIST)[6]. A atual revisão do PRODIST entrou em vigência em primeiro de janeiro de 2010. Dentre os principais objetivos do PRODIST encontra-se o de garantir que os sistemas de distribuição operem com segurança, eficiência, qualidade e confiabilidade. O Módulo 8 do PRODIST regula a qualidade da energia em redes de distribuição.

No âmbito da injeção dos harmônicos na rede elétrica, o PRODIST define os limites de correntes e tensões apresentados nas Tabelas I e II.

Apesar da legislação vigente não penalizar as indústrias pela geração de correntes harmônicas no sistema de energia, já existe indicação de que em breve a ANEEL divulgará uma nova legislação para avaliar os impactos oriundos das indústrias, conforme citação da nota técnica 0083/2012-SRD/ANEEL [7] onde a mesma faz referência a norma australiana que impõe valores de referência para a distorção harmônica da tensão (a serem observados pela distribuidora) e outros à corrente harmônica de equipamentos conectados na rede (a serem observados pelo consumidor).

Tabela I . Valores Globais Das Distorções Harmônicas Totais.

\begin{tabular}{|l|l|}
\hline $\begin{array}{l}\text { Tensão Nominal do } \\
\text { Barramento }\end{array}$ & $\begin{array}{l}\text { Distorção Harmônica } \\
\text { Total de Tensão (\%) }\end{array}$ \\
\hline $\mathrm{Vn} \leq 1 \mathrm{kV}$ & 10 \\
\hline $1 \mathrm{kV}<\mathrm{Vn} \leq 13,8 \mathrm{kV}$ & 8 \\
\hline $13,8 \mathrm{kV}<\mathrm{Vn} \leq 69 \mathrm{kV}$ & 6 \\
\hline $69 \mathrm{kV}<\mathrm{Vn} \leq 230 \mathrm{kV}$ & 3 \\
\hline
\end{tabular}

Tabela II. Níveis De Referência Para Distorções Harmônicas Individuais De Tensão.

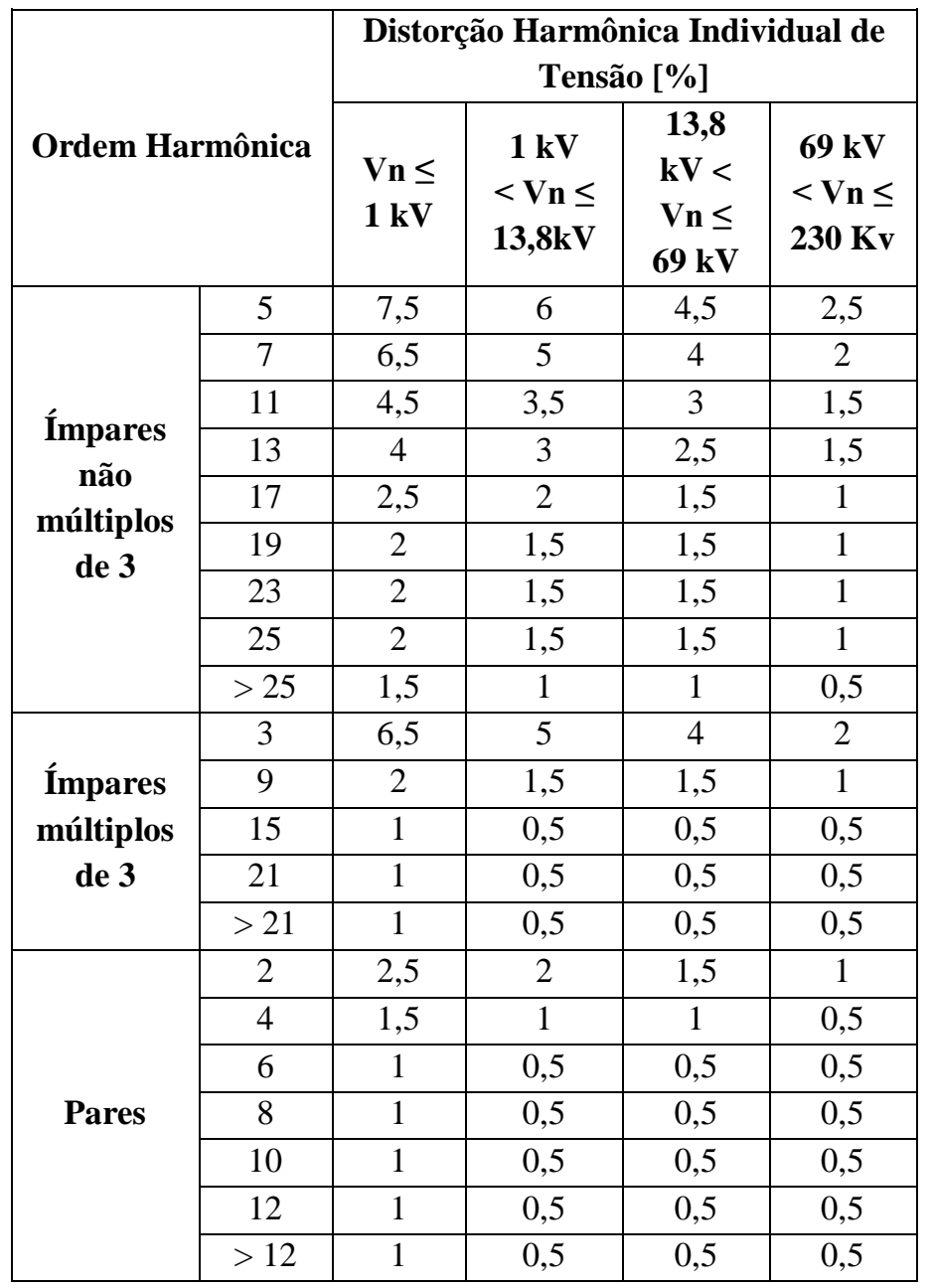

Apesar da legislação vigente não penalizar as indústrias pela geração de correntes harmônicas no sistema de energia, já existe indicação de que em breve a ANEEL divulgará uma nova legislação para avaliar os impactos oriundos das indústrias, conforme citação da nota técnica 0083/2012-SRD/ANEEL [7] onde a mesma faz referência a norma australiana que impõe valores de referência para a distorção harmônica da tensão (a serem observados pela distribuidora) e outros à corrente harmônica de equipamentos conectados na rede (a serem observados pelo consumidor).

Os efeitos das distorções harmônicas incluem: o aumento dos valores de pico de tensão (pode levar o sistema de isolamento à ruptura); o aumento dos valores eficazes de ondas alternada conhecida como RMS (Root Mean Square) ( leva a aumentar as perdas de potência) e o aumento da tensão e corrente de espectro de frequência (vibrações adicionais e desgaste mecânico) [8][9][10].

A circulação de correntes harmônicas produz aquecimento adicional em transformadores, cabos de alimentação, motores e alternadores, bem como avarias no sistema de correção do fator de potência. Além disso, problemas 
Rildo de Mendonça Nogueira, Edson Farias de Oliveira, et al / ITEGAM-JETIA Vol.01, Nº 01, pp.67-74. Março, 2015.

inesperados em dispositivos de proteção, operação errada em equipamentos eletrônicos sensíveis (computadores, equipamentos de comando e controle, etc.) e erros nos valores dos equipamentos de medição (por exemplo, balanças industriais eletrônicas, dosímetros eletrônicos, etc.).

Com o objetivo de avaliar os impactos das cargas não lineares em uma fábrica de computadores e permitir a antecipação com relação à legislação que em breve deverá surgir, foram definidas em conjunto com a indústria e os pesquisadores a metodologia a ser aplicada no referido trabalho: Instalar 5 medidores de qualidade de energia durante 7 dias em diferentes circuitos, coletar os dados, converter os dados, aplicar técnicas estatísticas, aplicar técnica de mineração de dados, aplicar inteligência computacional e avaliar os resultados.

Assim, esse estudo proporcionara uma análise através de árvore de decisão para identificar os impactos das componentes harmônicas individuais de corrente das cargas não lineares oriundas de equipamentos de informática nas distorções harmônica total de tensão - THDv de entrada de uma indústria montadora de equipamentos.

\section{MATERIAIS E MÉTODOS}

Optou-se pela metodologia KDD (Knowledge Discovery in Databases) ou Processo de Descoberta de Conhecimento em Base de Dados [11][12][13][14][15], pelo fato da mesma possuir características necessárias para a manipulação de grandes volumes de informações. Igualmente, o seu uso é justificável tendo em vista a necessidade de sistemas baseados em inteligência computacional.

No processo de descoberta de conhecimento em bases de dados, empregam-se várias etapas que se interligam como: Seleção, Pré-processamento, Transformação, Mineração de dados e Interpretação. Como demonstrado na Fig. 1.

Dentre as várias técnicas de mineração de dados utilizadas nas análises, selecionou-se a técnica de árvore de decisão [14][16][17]. A técnica selecionada foi definida por apresentar as seguintes características: um problema complexo é decomposto em subproblemas mais simples, recursivamente a mesma estratégia é aplicada em todos os subproblemas, Por levar em conta o atributo mais relevante na tomada de decisão, ser de mais fácil compreensão pela maioria das pessoas, escolher e representar os atributos por ordem de importância, ser de fácil apresentação em forma de árvore, considerando-se que as características apresentadas por esta técnica são aplicáveis para o estudo em questão, decidiu-se utilizá-la para a tomada de decisão.

De acordo com as etapas descritas no processo de descoberta do conhecimento KDD, adotou-se neste estudo o mesmo modelo de desenvolvimento.

- A etapa de Seleção

Os dados oriundos das medições foram coletados utilizando os analisadores de qualidade da energia elétrica (QEE) fornecida pela subestação para as linhas de produção de uma indústria do PIM, objeto deste estudo. Utilizou-se para este experimento cinco (5) analisadores de QEE para monitorar quatro (4) pontos nas saídas de energia elétrica, no chão de fábrica, e mais um (1) ponto monitorado no transformador que alimenta a subestação da indústria conforme exibe a Fig. 2 .

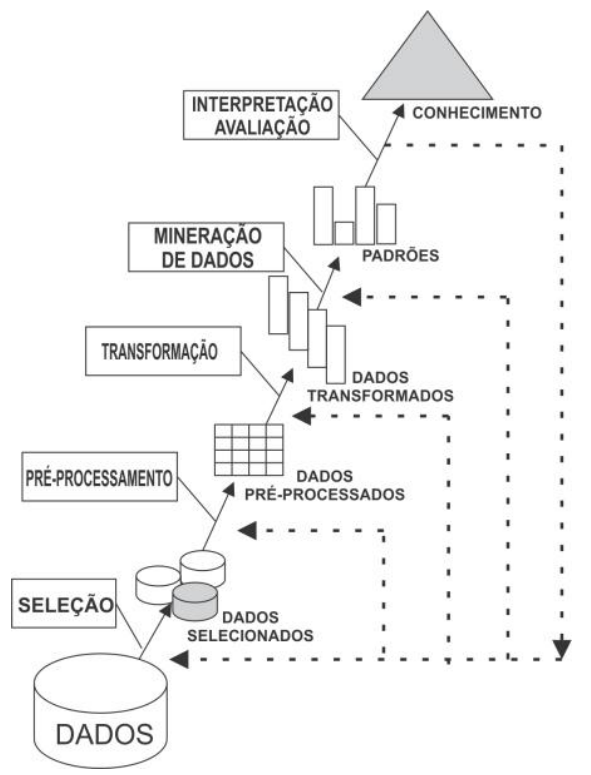

Figura 1. Processo de descoberta de conhecimento em bases de dado, (Adaptada de Fayyad et al., 1996)

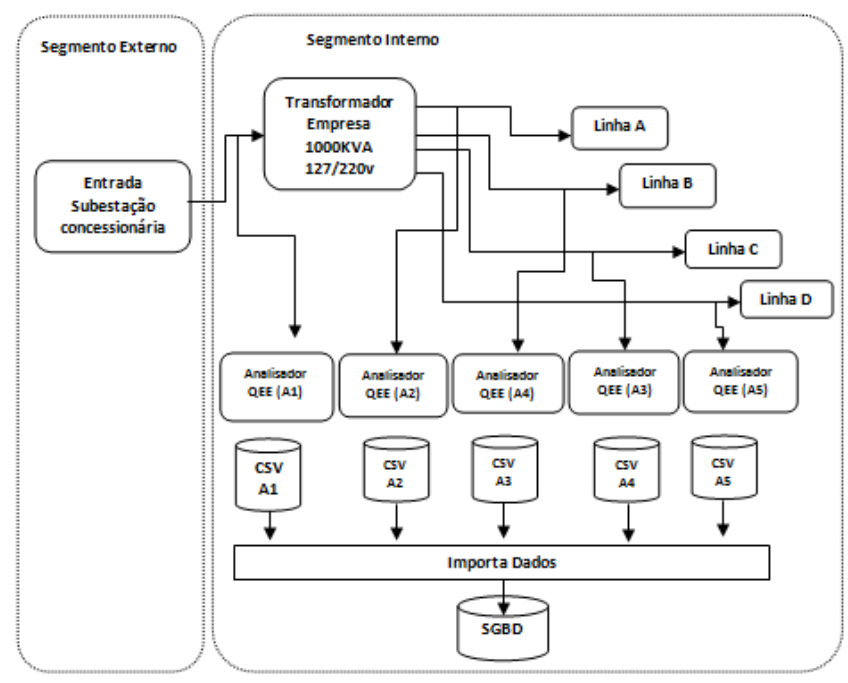

Figura 1. Modelo Conceitual do processo de Extração de dados

A massa de dados capturada pelos analisadores foi obtida no formato de texto, do tipo CSV (comma-separated values). Ao gerar os arquivos do tipo CSV, os dados inseridos neste arquivo são importados para um Sistema Gerenciador de Banco de Dados - SGBD, utilizando a ferramenta de importação de dados do Postgres [18], e por fim, armazená-los em tabelas respectivamente a cada característica de modelo de dados. A 
Rildo de Mendonça Nogueira, Edson Farias de Oliveira, et al / ITEGAM-JETIA Vol.01, Nº 01, pp.67-74. Março, 2015.

exemplo disto, os dados referentes à Fase A, foram coletados e armazenado na coluna thd_fase_a_entrada, do total de distorção Harmônica de tensão como demonstra a Tabela III.

Tabela III . Dados na base (sgbd)

\begin{tabular}{|c|c|c|c|c|c|}
\hline $\begin{array}{c}\text { Data } \\
\text { coleta }\end{array}$ & hora & $\begin{array}{c}\text { Thd } \\
\text { _fase_a } \\
\text { entrada }\end{array}$ & $\begin{array}{c}\text { H3_i_ } \\
\text { burning }\end{array}$ & $\begin{array}{c}\text { H5_i_- } \\
\text { burning }\end{array}$ & $\begin{array}{c}\text { H7_i_- } \\
\text { burning }\end{array}$ \\
\hline $2014-06-03$ & $13: 46: 00$ & 2.16 & 0.0750 & 0.0319 & 0.0312 \\
\hline $2014-06-03$ & $13: 47: 00$ & 2.24 & 0.0743 & 0.0317 & 0.0310 \\
\hline $2014-06-03$ & $13: 48: 00$ & 2.27 & 0.0749 & 0.0321 & 0.0314 \\
\hline
\end{tabular}

As variáveis analisadas, pertinentes às harmônicas de corrente, são de ordem $3^{\circ}, 5^{\circ}, 7^{\circ}, 9^{\circ}$ e $11^{\circ}$ no ambiente fabril; e na entrada do transformador selecionou-se a Distorção Harmônica Total de tensão (Total Harmonic Distortion) THDv em percentual[1]. As harmônicas causadoras dos maiores impactos nas redes elétricas são de ordem $3^{\circ}, 5^{\circ}$ e $7^{\circ}$.

As outras variáveis que compõem este estudo representam: os turnos (representando os diversos turnos em que o ambiente fabril está em atividade e suas respectivas folgas) e o momento de cada registro realizado pelos analisadores de QEE (data e hora da ocorrência).

\section{- A etapa de Pré-processamento}

Depois da coleta dos dados na etapa de Seleção, os resultados obtidos deste processo são submetidos a fase Préprocessamento onde são realizados os processos de filtragens, para eliminação de dados redundantes, inconsistentes e recuperação de dados incompletos, nesta etapa também são utilizados métodos de redução e transformação para diminuir a quantidade de variáveis, visando com isto melhorar o desempenho do algoritmo que será utilizado na análise. Nessa fase executou-se a discretização dos dados contínuos, com objetivo de transforma-los em intervalos ou conjuntos de dados menores e mais fáceis de serem analisas. $\mathrm{O}$ filtro executado foi de discretização não supervisionada com Cinco (5) faixas por equalização dos dados por frequência para todos os atributos analisados.

- A etapa de Transformação dos dados

Após os processos de aquisição, limpeza, filtragens os dados são transformados em formatos utilizáveis, navegáveis. Pelo processo de aprendizagem de acordo com o algoritmo escolhido na fase seguinte.

- A etapa de Mineração de dados (Dataming)
Através do emprego da técnica de classificação por árvore de decisão será possível gerar conhecimentos para auxiliar na tomada de decisão sobre as análises das harmônicas que estão causando maiores impactos na rede elétrica da indústria.

A tabela IV demonstra o conhecimento originado pela aplicação da técnica de árvore de decisão sobre uma base de dados, empregando o algoritmo C4. 5 [14] que em alguns softwares de Inteligência Computacional é conhecido por J48. Os resultado apresentados foram implementado no software de mineração de dados WEKA [19].

Tabela IV. Dados algoritmo j48 no WEKA

\begin{tabular}{|c|c|}
\hline Dados Analisados & Resultados Obtidos \\
\hline $\begin{array}{ll}\text { Instâncias } & \text { corretamente } \\
\text { classificados } & \end{array}$ & 2747 com $90.6601 \%$ \\
\hline $\begin{array}{l}\text { Instâncias incorretamente } \\
\text { classificados }\end{array}$ & $283 \operatorname{com} 9.3399 \%$ \\
\hline Estatística Kappa & 0,8832 \\
\hline A média de erro absoluto & 0,0595 \\
\hline $\begin{array}{l}\text { K \& B Informação Relativa } \\
\text { Pontuação }\end{array}$ & $260664.6525 \%$ \\
\hline 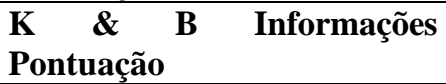 & $\begin{array}{l}6051.977 \text { bits } \\
1,9974 \text { bits / exemplo }\end{array}$ \\
\hline $\begin{array}{l}\text { Complexidade da Classe I } \\
\text { ordem } 0\end{array}$ & $\begin{array}{l}7034.8954 \text { bits } \\
2,3217 \text { bits / instância }\end{array}$ \\
\hline $\begin{array}{l}\text { Complexidade da Classe I } \\
\text { esquema }\end{array}$ & $\begin{array}{l}1109.2512 \text { bits } \\
0,3661 \text { bits / instância }\end{array}$ \\
\hline Melhoria Complexidade (sf) & $\begin{array}{l}5925,6442 \text { bits } \\
1,9557 \text { bits / instância }\end{array}$ \\
\hline $\begin{array}{lccc}\text { Raiz do } & \text { erro } & \text { médio } \\
\text { quadrado } & & \\
\end{array}$ & 0,1725 \\
\hline Erro Relativo absoluto & $18,599 \%$ \\
\hline Raiz relativa erro quadrado & $43,126 \%$ \\
\hline $\begin{array}{l}\text { Cobertura dos casos }(0,95 \\
\text { nível) }\end{array}$ & $99,571 \%$ \\
\hline $\begin{array}{l}\text { Rel. média. Tamanho da } \\
\text { região }(0,95 \text { nível) }\end{array}$ & $31,7624 \%$ \\
\hline Número total de instâncias & 3030 \\
\hline
\end{tabular}

Os softwares de inteligência computacional utilizados na mineração dos dados e nas análises e tomada de decisões foram WEKA 3.7[19] e RapidMiner 5[20].

\section{- Interpretação e Avaliação}

Após a identificação dos padrões, o sistema interpreta os dados resultantes do processo do KDD, transforma-os em conhecimento que por sua vez darão suporte a tomada de decisões, muitas decisões são tomadas a partir do conhecimento do especialista. 
Rildo de Mendonça Nogueira, Edson Farias de Oliveira, et al / ITEGAM-JETIA Vol.01, Nº 01, pp.67-74. Março, 2015.

\section{INDÚSTRIA DE MANUFATURA DE PRODUTOS ELETRO-ELETRONICOS (COMPUTADORES) - ESTUDO DE CASO}

O referido trabalho foi desenvolvido a partir da coleta de dados em uma Indústria de manufatura de produtos eletroeletrônicos instalada no Polo Industrial de Manaus - PIM.

Com a parceria firmada com a Indústria de Produtos Manufaturados que possuía objetivos de anteciparem-se à nova legislação que irá avaliar os impactos das correntes harmônicas oriundas das indústrias e também o de melhorar os seus indicadores de qualidade de energia, foram feitas as coletas dos dados.

A característica principal é que a fábrica em análise monta computadores pessoais, monitores de computadores, notebooks e tablets. Produtos estes que são geradores de distorções harmônicas.

Durante a coleta dos dados, a fábrica trabalhava em 2 turnos de segunda a sexta, sendo o primeiro turno de 06:00 às 15:48 e segundo de 16:00 às 01:30. Durante esse período a mesma estava produzindo notebooks e tablets.

Foram escolhidos para a coleta de dados os seguintes pontos: saída do quadro de alimentação $(220 / 127 \mathrm{~V})$, circuito que alimenta as centrais de ar condicionado, circuito que alimenta a sala de compressores, circuito que alimenta a linha de teste de notebook, com aproximadamente 900 notebooks ligados simultaneamente, circuito que alimenta a sala de montagem de tablets, com aproximadamente 140 tablets ligados simultaneamente.

Nestes pontos, foram instalados 5 medidores de qualidade de energia HIOKI PW3198, conforme está evidenciado na Figura 3.

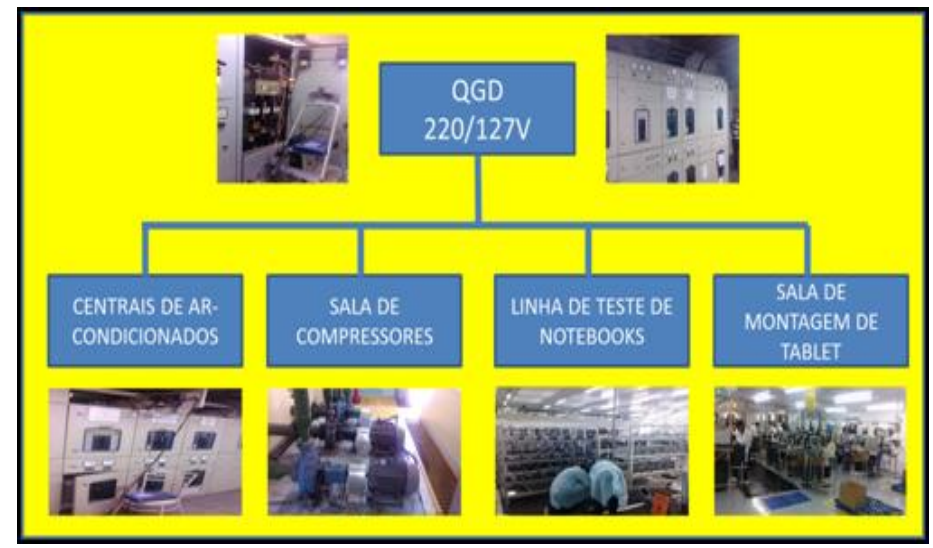

Figura 3. Pontos de Instalações dos Medidores de Qualidade Energia Elétrica

As Tabelas V, VI, VII, VIII e IX descrevem os detalhes dos quadros de alimentacão e as características técnicas e de geração de harmônicas de cada circuito, os tipos de cargas, bem como, Os detalhes dos períodos que permaneceram alimentados.
Tabela V. Quadro Geral da Subestação:

\begin{tabular}{|c|c|c|}
\hline \multicolumn{3}{|l|}{ Cargas } \\
\hline Origem & TDHi & Harmônicas \\
\hline Fonte Chaveada & $>93 \%$ & $\begin{array}{l}3(78 \%) \\
7(17 \%) \ldots 15\end{array}$ \\
\hline $\begin{array}{l}\text { Inversores } \\
\text { frequência }\end{array}$ & $\begin{array}{l}> \\
100 \%\end{array}$ & $\begin{array}{l}5(81 \%), 7(74 \%), 11(42 \%), 1 \\
3(30 \%), 17 \text { e } 19\end{array}$ \\
\hline UPS & $>30 \%$ & $\begin{array}{l}5(28 \%), 7(5 \%), 11(6 \%) \mathrm{e} \\
13(6 \%)\end{array}$ \\
\hline $\begin{array}{l}\text { Lâmpadas } \\
\text { Fluorescente }\end{array}$ & $>50 \%$ & $3,5,9$ e 11 \\
\hline
\end{tabular}

Tabela VI. Centrais de ar-condicionado

\begin{tabular}{|l|l|}
\hline Cargas & $\begin{array}{l}\text { 04 Centrais de Ar-condicionado. } \\
\text { Cada Central funciona com 2 compressores } \\
\text { de 15TR }(52,75 \mathrm{~kW})\end{array}$ \\
\hline $\begin{array}{l}\text { Características } \\
\text { Harmônicas }\end{array}$ & $\begin{array}{l}\text { Circuito de Carga Linear com baixo impacto } \\
\text { na distorção harmônica }\end{array}$ \\
\hline $\begin{array}{l}\text { Comentários } \\
\text { sobre } \\
\text { funcionamento }\end{array}$ & $\begin{array}{l}\text { São ligados entre 05:30 até 01:30 de } \\
\text { segunda a sexta. }\end{array}$ \\
\hline
\end{tabular}

Tabela VII. Linha burning test

\begin{tabular}{|c|c|}
\hline Cargas & $\begin{array}{l}\text { Em torno de } 500 \text { notebook de } 65 \mathrm{~W} \text { cada } \\
\text { bifásico } \\
\text { Especificação da fonte chaveada de } 65 \mathrm{~W} \\
\text { informa: } \\
\text { Corrente de Carga Nominal: } 3,42 \mathrm{~A} \\
\text { Potência de Saída Nominal : } 65 \mathrm{~W} \\
\text { Regulação de Saída Total: +/- } 5 \% \\
\text { Regulação de Linha: +- } 3 \% \\
\text { Ripple: } 200 \mathrm{mV} \text { (pico a pico) } \\
\text { Ruído: } 380 \mathrm{mV} \text { (pico a pico) } \\
\text { THD, V }<2 \%\end{array}$ \\
\hline $\begin{array}{l}\text { Característic } \\
\text { as } \\
\text { Harmônicas }\end{array}$ & $\begin{array}{l}\text { Carga oriunda de Fonte Chaveada. } \\
\text { THDi > 93\%. } \\
\text { Harmônicas: } 3 \text { (78\%), } 5(44 \%), 7(17 \%) \ldots 15 \\
\text { A linha estava ligada em UPS de 80kVA e } \\
\text { cuja característica é: } \\
\text { TDHi > 30\% } \\
\text { Harmônicas: } 5 \text { (28\%), } 7(5 \%), 11(6 \%) \text { e } 13 \\
(6 \%)\end{array}$ \\
\hline $\begin{array}{l}\text { Comentários } \\
\text { sobre o } \\
\text { funcionamen } \\
\text { to }\end{array}$ & $\begin{array}{l}\text { Cada notebook fica ligado por } 1 \text { hora e é } \\
\text { substituído por outro. } \\
\text { No período de 01:30 às 06:00 ficam ligados os } \\
\text { notebook do dia anterior. Durante o final de } \\
\text { semana os notebooks ficam ligados na rede } \\
\text { elétrica. Todos os notebooks são alimentados } \\
\text { por um No-Break (UPS) de } 80 \mathrm{kVA} \text {. }\end{array}$ \\
\hline
\end{tabular}




\begin{tabular}{|c|c|c|}
\hline $\begin{array}{l}\text { mi } \\
0 \\
0\end{array}$ & \multicolumn{2}{|c|}{$\begin{array}{c}\text { Rildo de Mendonça Nogueira, Edson Farias de Oliveira, e } \\
\text { Tabela VIII. Produção De Tablets }\end{array}$} \\
\hline 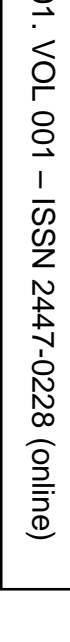 & Cargas & $\begin{array}{l}\text { 10 Impressoras Laser com } 57,96 \mathrm{~W} \text { cada } \\
4 \text { Balanças Eletrônicas com } 15 \mathrm{~W} \text { cada } \\
10 \text { parafusadeiras elétricas com motor de } \\
40 \mathrm{~W} \text { cada } \\
11 \text { switch de rede com } 10,3 \mathrm{~W} \text { cada } \\
4 \text { Monitores de LED com } 45 \mathrm{~W} \text { cada } \\
1 \text { Computador DeskTop de } 352 \mathrm{~W} \\
1 \text { Ferro de Soldar de } 70 \mathrm{~W} \\
3 \text { Ionizador de Ar com } 26,4 \mathrm{~W} \text { cada } \\
8 \text { impressora de etiqueta com } 110 \mathrm{~W} \text { cada } \\
17 \text { notebooks com } 65 \mathrm{~W} \text { cada } \\
25 \text { lâmpadas LED com } 20 \mathrm{~W} \text { cada } \\
12 \text { dispensador eletrônico de parafusos com } \\
6 \mathrm{~W} \text { cada } \\
\text { Em torno de } 140 \text { tablet ligados em teste com } \\
10 \mathrm{~W} \text { cada }\end{array}$ \\
\hline & $\begin{array}{l}\text { Características } \\
\text { Harmônicas }\end{array}$ & $\begin{array}{l}\text { A maioria das cargas são oriundas de Fonte } \\
\text { Chaveada. } \\
\text { THDi > 93\%. } \\
\text { Harmônicas: } 3 \text { (78\%), } 5 \text { (44\%), } 7 \text { (17\%) ... } \\
15 \\
\text { Existindo também as parafusadeiras elétricas } \\
\text { com as seguintes características harmônicas: } \\
\text { THDi > 100\%. } \\
\text { Harmônicas: } 5 \text { (81\%), } 7 \text { (74\%), } 11 \text { (42\%), } \\
13(30 \%), 17 \text { e } 19 \\
\text { A linha estava ligada em UPS de 80kVA e } \\
\text { cuja característica é: } \\
\text { TDHi > 30\% } \\
\text { Harmônicas: } 5 \text { (28\%), } 7 \text { (5\%), 11(6\%) e } 13 \\
(6 \%)\end{array}$ \\
\hline & $\begin{array}{l}\text { Comentários } \\
\text { sobre o } \\
\text { funcionamento }\end{array}$ & $\begin{array}{l}\text { A Sala funciona de 06:00 às } 01: 30 \text { do dia } \\
\text { seguinte de segunda a sexta, onde os } \\
\text { equipamentos da linha são desligados. } \\
\text { No período de } 01: 30 \text { a } 06: 00 \text { ficam alguns } \\
\text { tablets ligados. } \\
\text { Todos os equipamentos e tablets são } \\
\text { alimentados por um No-Break (UPS) de } \\
\text { 80kVA. }\end{array}$ \\
\hline
\end{tabular}

Tabela IX. Compressores e Bombas

\begin{tabular}{|l|l|}
\hline \multirow{2}{*}{ Cargas } & $\begin{array}{l}\text { 1 Compressor de 75 CV }(55,93 \mathrm{~kW}) \\
\text { 1 Bomba de Recalque de 25 CV }(18,64 \mathrm{~kW}) \\
\text { 1 Bomba de Torre de 25 CV }(18,64 \mathrm{~kW})\end{array}$ \\
\hline $\begin{array}{l}\text { Características } \\
\text { Harmônicas }\end{array}$ & $\begin{array}{l}\text { Circuito de Carga Linear com baixo impacto } \\
\text { na distorção harmônica }\end{array}$ \\
\hline $\begin{array}{l}\text { Comentários } \\
\text { sobre } \\
\text { funcionamento }\end{array}$ & $\begin{array}{l}\text { São ligados entre 05:30 até 01:30 de segunda } \\
\text { a sexta. }\end{array}$ \\
\hline
\end{tabular}

\section{ANÁLISE DOS DADOS}

No presente estudo, buscou-se avaliar como as correntes harmônica de ordem 3, 5, 7, 9 e 11 provenientes dos processos da indústria analisada: burning, tablets, compressor e centrais de ar, influenciam na taxa de distorção harmônica total de tensão (THDv) da subestação de entrada da indústria. Para tal, construiu-se, por meio do algoritmo C4.5, uma árvore de decisão que relaciona as correntes harmônicas de ordem 3, 5, 7, 9 e 11 com o THDv do secundário do transformador de entrada da subestação da indústria, cujo nível de tensão é $220 \mathrm{~V}$.

A árvore de decisão criada apresentou como nó raiz a corrente do $7^{\circ}$ harmônico do processo de tablets, o que indica que essa corrente harmônica apresenta uma correlação linear significativa com o THDv da entrada da indústria. Em virtude da árvore de decisão criada pelo software de mineração de dados ser bastante grande, escolheu-se três sub-árvores que são capazes de generalizar o conhecimento adquirido com o uso da técnica empregada na avaliação dos dados.

Analisando a sub-árvore 1, Fig. 5, pode-se observar que, tanto no segundo turno quanto no intervalo da madrugada, o valor THDv se manterá entre 2,705 e $3,085 \%$ se a corrente do $7^{\circ}$ harmônico se mantiver entre 3,75 e $4,85 \%$, ou seja, praticamente somente a corrente do $7^{\circ}$ harmônico do processo de tablets seria o suficiente para obter o valor do THDv, porém o mesmo não aumentaria para valores acima de $4,85 \%$ ou diminuiria para valores abaixo de $3,75 \%$.

Durante o $1^{\circ}$ turno, se a corrente do $7^{\circ}$ harmônico do processo de tablets estiver, entre 3,75 e $4,85 \%$, o valor do THDv de tensão dependerá do dia da semana, e da corrente do $3^{\circ}$ harmônico do processo de tablets ou do $5^{\circ}$ harmônico do processo centrais de ar.

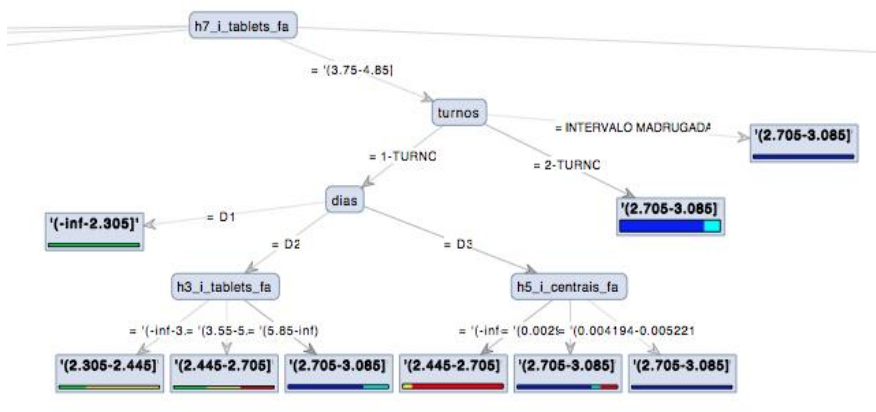

Figura 4. Sub árvore 1 (fase A)

Analisando a sub-árvore 2 Fig. 6, pode-se observar que, caso o valor da corrente do $7^{\circ}$ harmônico do processo de tablets apresente um valor entre 3,35 e 3,75\%, durante o primeiro turno, o valor do THDv no secundário do transformador de entrada da industria dependerá do dia da semana e da corrente do $7^{\circ}$ harmônico do processo centrais de ar. No entanto, durante o 
Rildo de Mendonça Nogueira, Edson Farias de Oliveira, et al / ITEGAM-JETIA Vol.01, N ${ }^{\mathrm{o}}$ 01, pp.67-74. Março, 2015.

segundo turno, o THDv dependerá da corrente do $7^{\circ}$ harmônico do processo centrais de ar e da corrente do $5^{\circ}$ harmônico do processo compressor. Além disso, deve-se notar que nessa subárvore, o mínimo e máximo valor que o THDv atingirá é 2,305\% e $3,085 \%$, respectivamente.

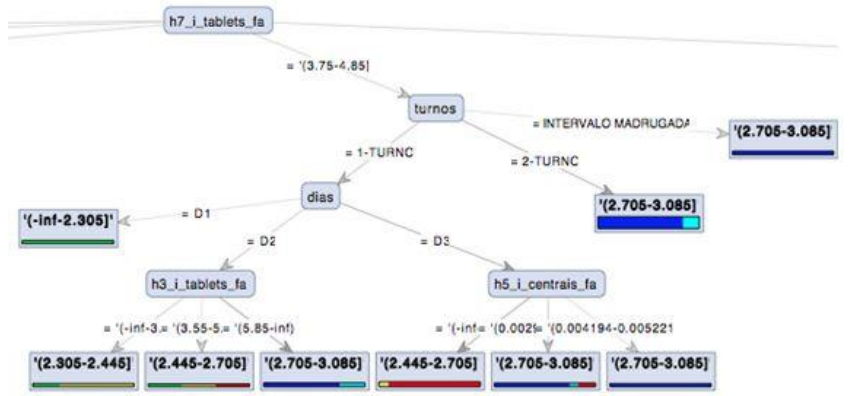

Figura 5. Sub Árvore 2 (fase A)

Analisando a sub-árvore 3, Fig. 7, pode-se verificar que, se a corrente do $7^{\circ}$ harmônico do processo de tablets for maior que $4,85 \%$ e a corrente do $5^{\circ}$ harmônico do processo de tablets for maior que $3,05 \%$, o THDv apresentara um valor igual ou maior que $3,085 \%$. Logo, o aumento do THDv no secundário do transformador de entrada da subestação da industria está fortemente relacionado com o aumento das correntes do $7^{\circ}$ e $5^{\circ}$ harmônicos, conforme pode ser visualizado na Fig. 3. Além disso, caso a corrente do $5^{\circ}$ harmônico do processo de tablets se mantiver entre 1,65 e $3,05 \%$, a corrente do $5^{\circ}$ e $7^{\circ}$ harmônico do processo de burning propiciarão o aumento do THDv para níveis acima de $3,085 \%$.

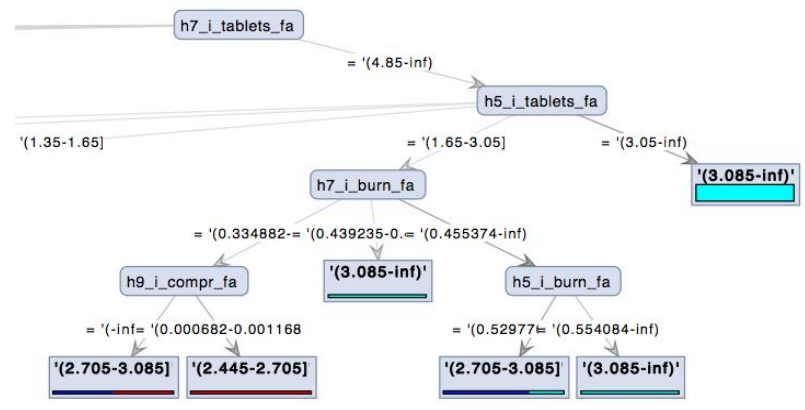

Figura 6. Sub Árvore 3 (fase A)

\section{CONCLUSÃO}

Considerando os aspectos analisados no experimento entende-se que a aplicação da técnica de árvore de decisão resultou que os impactos mais significativos foram detectados nas harmônicas de $5^{\mathrm{a}}$ e $7^{\mathrm{a}}$ ordem devido às características do processo desenvolvido pela linha de tablets que apresentou a $7^{\mathrm{a}}$ harmônica sendo a de maior impacto de distorções no processo fabril da indústria. De acordo o PRODIST [9] no módulo 8, e a norma IEEE[21], que regulamentam os níveis mínimos e máximos de distorções harmônicas nas redes elétricas, a $7^{\mathrm{a}}$ harmônica da linha de tablets apresentou os níveis mais elevados de distorções harmônicas em vários períodos e datas diferentes no decorrer da execução do seu processo. Os demais processos da indústria tiveram uma baixa geração ou quase nenhuma geração de impactos de harmônicos na rede elétrica.

\section{AGRADECIMENTOS}

A Universidade Federal do Pará - UFPA, ao Instituto de Tecnologia Galileo da Amazônia - ITEGAM e ao Instituto de Tecnologia José Rocha Sérgio Cardoso - ITJC, Instituto Certi da Amazônia - ICA. Eletrobrás Amazonas Energia pelo apoio à pesquisa.

\section{REFERÊNCIAS}

[1] M.E.L. Tostes, "Avaliação dos impactos causados pela geração de harmônicos na rede de distribuição em Consumidores em baixa tensão". Tese de Doutorado do Programa de Pós-Graduação em Engenharia Elétrica do Instituto de Tecnologia da Universidade Federal do Pará (ITEC-UFPA). 2003.

[2] C.F. Nascimento, A.A. Oliveira Jr., A. Goedtel, P.J.A. Serni, Harmonic identification using parallel neural networks in single-phase systems, Applied Soft Computing 11 (2) (2011) 2178-2185.

[3] A. Ferrero, Measuring electric power quality: problems and perspectives, Measurement 41 (2) (2008) 121-129.

[4] T. Tarasiuk, Estimator-analyzer of power quality. Part I. Methods and algorithms, Measurement 44 (1) (2011) 238-247.

[5] S. Vlahinic, D. Brnobic, N. Stojkovic, Indices for harmonic distortion monitoring of power distribution systems, IEEE Transactions on Instrumentation and Measurement 58 (5) (2009) 1771-1777.

[6] ANEEL. Módulo 8 - Procedimentos de Distribuição de Energia Elétrica no Sistema Elétrico Nacional - PRODIST. 2010.

[7] ANEEL. Nota Técnica, $\mathbf{n}^{\circ} 0083$ /2012-SRD/ANEEL, de 12 Junho 2012. Processo: 48500.002798/2012-61. 
Rildo de Mendonça Nogueira, Edson Farias de Oliveira, et al / ITEGAM-JETIA Vol.01, Nº 01, pp.67-74. Março, 2015.

[8] R. Dugan, M. McGranaghan, H. Wayne, "Electrical Power Systems Quality", 265 pp., McGraw-Hill, New York, USA. 1999.

[9] IEEE Task Force on Effects of Harmonics on Equipment: "Effects of Harmonics on Equipment", IEEE Transactions on Power Delivery, vol. 8, n. ${ }^{\circ}$ 2, pp. 672-680, April 1993.

[10] UIE, "Types of Disturbances and Relevant Standards, Guide to Quality of Electrical Supply for Industrial Installations", "Power Quality" Working Group WG2, 1996.

[11] Quinlan, J. R. C4.5: Programs for Machine Learning. Morgan Kaufmann Publishers, 1993.

[12] FAYYAD, U.; PIATETSKY-SHAPIRO, G.; SMYTH, $P$. From data mining to knowledge discovery in databases. AI magazine, [s.1], v. 17, p. 37-54. 1996.

[13] Data mining techniques and applications - A decade review from 2000 to 2011. Review Article Expert Systems with Applications, Volume 39, Issue 12, 15 September 2012, Pages 11303-11311 Shu-Hsien Liao, Pei-Hui Chu, Pei-Yuan Hsiao.

[14] NEVES, R. de C. D das. Pré-processamento no processo de descoberta de conhecimento em banco de dados. 2003. Dissertação (Programa de Pós-graduação em Computação) - Instituto de Informática, Universidade Federal do Rio Grande do Sul, Porto Alegre, 2003.

[15] Data mining techniques and applications - A decade review from 2000 to 2011. Review Article Expert Systems with Applications, Volume 39, Issue 12, 15 September 2012, Pages 11303-11311 Shu-Hsien Liao, Pei-Hui Chu, Pei-Yuan Hsiao

[16] Knowledge representation and acquisition approach based on decision tree Jianshe Bai ; Bo Fan ; Junyi Xue; Natural Language Processing and Knowledge Engineering, 2003. Proceedings. 2003 International Conference on DOI: 10.1109/NLPKE.2003.1275962 Publication Year: 2003 , Page(s): $533 \quad-538$

[17] CARVALHO, L, A. V. de. Datamining: a mineração de dados no Marketing, Medicina, Economia, Engenharia e Administração. São Paulo: Ciência Moderna,2006.

[18] PostgreSQL: The world's most advanced open source The PostgreSQL Global Development Group is pleased to announce the availability of PostgreSQL 9.4 Beta 2, the second beta release of the upcoming 9.4. Disponível em:< www.postgresql.org/> Acesso em: 07 Julho 2014.

[19] UNIVERSITY OF WAIKATO. Weka 3 - Machine Learning Software in Java. Disponível no site da University of Waikato (2010). URL: http://www.cs.waikato.ac.nz/ml/weka.

[20] Rapid Miner. Provides software, solutions, and services in the field of advanced analytics, including predictive analytics, data mining, and text mining. URL: http://http://rapidminer.com/

[21] IEEE Std. 519-1992. IEEE Recommended Practices and Requirements for Harmonic Control in Electrical Power Systems. 1992. 